\title{
Comparative Aesthetics of Chinese and Western Visual Poetry Based on the Multimodal Theories
}

\author{
XU Ming, ZHANG Qing \\ China University of Political Science and Law, Beijing, China
}

\begin{abstract}
Although the Chinese visual poetry and western visual poetry have a lot of differences, the similarity of visual poetry is the combination of poetry and painting. This research will apply the method of comparative analysis and multimodal theories, aiming to provide a new view to evaluate Chinese and western poetry, so as to promote the aesthetic experiences of Chinese and western culture for public. The research finds that Chinese and western poetry can be analyzed from the perspective of framework of visual-image analysis based on multimodal theories, which is composed of three major meanings: representation, interaction and composition. The comparative aesthetics based on the multimodal theories is of great significance to the appreciation and enjoyment of Chinese and western visual poetry.
\end{abstract}

Keywords: visual poetry, comparative aesthetics, multimodal theories, constructivist, expressionist

\section{Introduction}

As a type of literature and culture, Chinese and western visual poetry are received more and more attention. People may have different perspectives towards visual poetry. This research applies the method of comparative analysis which have been done before, by providing a contrast of traditional analysis and the multimodal theories based analysis on Chinese visual poetry and western visual poetry. This research aims to provide a new view to evaluate Chinese and western poetry, that is, using representation, interaction and composition to analyze the poetry, so as to promote the aesthetic experiences of Chinese and western culture for public. The structure of the research will begin with the theoretical introduction, and then it will focus on the three materials, Chinese visual poetry, western visual poetry and the visual poetry created by the author, which will apply the comparative methods in the process of analysis, and finally, it will be the conclusion part.

Scholars from the domestic and abroad provide more views about visual poetry. Some researches have been done from different perspectives. Tan (2006) of Indiana University has made an analysis on the definition of visual poetry, the contrast of traditional visual poetry and modern visual poetry and the appreciation of visual poetry from the theoretical perspective. $\mathrm{Li}$ (2010) has taken an example of analyzing the visual poetry "40-Love" written by Roger McGough based on the multimodal discourse analysis, illustrating that the systemic functional grammar has strong superiority in the analysis of visual poetry. Jin (2012) also gives an example of the visual

XU Ming, postgraduate of School of Foreign Languages, China University of Political Science and Law, Beijing, China. ZHANG Qing, Professor, China University of Political Science and Law, Beijing, China. 
poetry "in Just" based on the multimodal discourse analysis, holding that the symbols, images and other symbolic systems in "in Just" complement each other and serve as an correspondence for each other, resulting in multimodal effects. Though there are relevant researches about visual poetry, however, those two latter scholars didn't analyze the texts in different languages. Therefore, this research makes an innovation in comparing the traditional analysis method and the multimodal theories based method to analyze the Chinese visual poetry and western visual poetry, besides, adding the visual poetry created by the author of this paper. Therefore, the methods are different and the textual materials are richer and more innovative.

\section{The Theoretical Basis on Visual Poetry and Multimodal Discourse Analysis}

The theoretical basis of this research is the traditional analysis based theories and the multimodal theories. Before introducing the multimodal discourse analysis, in order to make analysis clearly, the present part will introduce the visual poetry firstly.

\section{The Theoretical Basis of Visual Poetry}

Visual poetry can be regarded as a poetry meant to be seen. Combining painting and poetry, it is neither a compromise nor an evasion but a synthesis of the principles underlying each medium. In its own way it is one of the most radical inventions (or reinventions) of our time (Bohn, 1986). Tan (2006) holds that visual poetry has two main features. Visual poetry breaks the habit of appreciation of ordinary people, synchronizing the moving lines with the static visual images. The shape of the image becomes the basis for the content. Just as the meanings of vocabularies depend on the constructive forms in some aspects, the meanings of visual poetry depend on the arrangement and composition of vocabularies in space. Another feature of visual poetry is its untranslatability. As vocabularies are the part of the composition in visual poetry, any changes to it will affect the whole image. This is a major reason why the spread of visual poetry is limited.

The constructivist poem results from an arrangement of materials according to a schema or system set up by the poet which must be adhered to on its own terms (permutational poems). In the expressionist poem the poet arranges his material according to an intuitive structure (Solt, 1968).

\section{The Theoretical Basis of Multimodal Discourse Analysis}

Modal refers to the channels and media of communication, including language, technology, image, color, music and other symbolic systems (Zhu, 2007). Foreign scholars Kress and Van Leeuwen (2006) define multimodality as an interdisciplinary research hotspot, which refers to the language, text, print and digital media, specific actions and 3D things and places via the occurrence of social semiosis. Li (2003), a domestic scholar, believes that multimodality refers to any text that is more than one type of code-compatible meanings, such as images and charts, in addition to the text itself. The multimodal discourse analysis is to analyze the discourses in multimodal texts that integrate all kinds of modals, such as character, color, image, sound and so forth.

The theory of multimodal discourse analysis is the metafunctions of language put forward by Halliday, including ideational function, interpersonal function and textual function. Ideational function refers to the fact that language has the function to convey new information, to communicate a content that is known to the hearer. Interpersonal function refers to the fact that language has the function to embody all uses of language to express social and personal relations. This includes the various ways the speaker enters a speech situation and performs a 
speech act. Textual function refers to the fact that language has mechanisms to make any stretch of spoken and written discourse into a coherent and unified text and make a living passage different from a random list of sentences.

\section{The Analysis of Chinese and Western Visual Poetry}

On the basis of multimodal discourse analysis, Kress and Van Leeuwen (2006) compare the three functions of the systematic functional theory of Halliday, and establish the framework of visual-image analysis, which is composed of three major meanings: representation, interaction and composition. Among them, representational metafunction corresponds to conceptual function, including narrative representation and conceptual representation; interactive metafunction corresponds to interpersonal function, related to three elements and modality design, distance, contact and point of view; the compositional metafunction is equivalent to textual function, from the three aspects of information value, shooting a scene and prominence (Wang, 2009).

This present part will analyze the Chinese visual poetry and western visual poetry from part to whole. By focusing on the three dimensions of multimodal theories, it attempts to establish a new framework to analyze visual poetry. As we dissect the multimodal theories based on the systematic functional theory, instead of the three original functions, the new three-dimensional structure is representational meaning, interactive meaning and compositional meaning. The analysis here will draw heavily on the different kinds of materials applying the new framework: representation, interaction and composition.

\section{The Analysis of Western Visual Poetry}

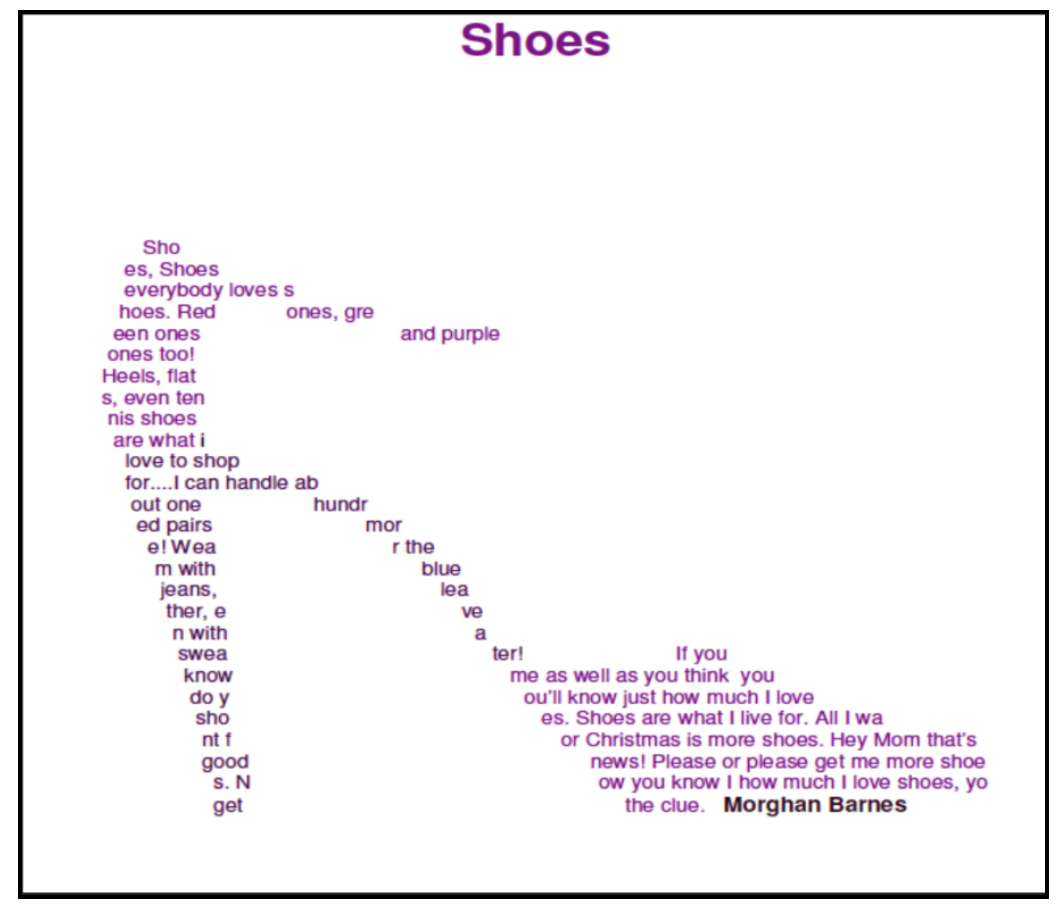

Figure 1. Shoes.

Firstly, the analysis focuses on the traditional perspectives. In the visual image, it is a shape of a shoe, specifically, a high-heeled shoe. There are two colors in this visual poetry, deep pink and dark pink. Because the 
warm tone of colors, such as pink, red, orange, usually serves for women, the deep pink and dark pink mean that the shoes are for women. There are also several vocabularies about color in this visual poetry, such as "red", "green", "purple", which give readers an impression of colors in minds. There are several types of shoes that the poet mentions, such as "heels", "flats", "tennis shoes", broadening the readers' impressions of shoes rather than limiting to the shape of the high-heeled shoe in this poetry. The word "heels" is rightly in the position of heels. Besides, due to the beginning of a new sentence, the word "heels" is capitalized. All of these phenomena in the poetry make a dialogue between visual images and vocabularies. By describing the woman's desperate desire for shoes in the poetry, the poet expresses women's pursuit of beauty.

Secondly, from the perspective of multimodal discourse analysis based on the systematic functional theories, the analysis can be developed from three dimensions, that is, representation, interaction and composition. (1) Representational metafunction, as mentioned above, includes narrative representation and conceptual representation, which works mainly through the typographical device. By the typographical device and the way of white space, a shape of woman's high-heeled shoe is in sight immediately. The shape represents the concept of woman's high-heeled shoe on written texts, and at the same time, represents an image of woman's high-heeled shoe in readers' minds. (2) Interactive metafunction is about the relationship between the poet, the indicated things (including people) and the readers of the images, as well as the attitude of the readers to the indicated things. Images can cause a specific relationship between the readers and the world in the images. They interact with the readers and give a hint of the readers' attitude to the indicated scene $(\mathrm{Li}, 2003)$. There are three elements to achieve this interactive metafunction: distance, point of view and contact. The shape in this visual poetry is represented in the way of close shot from the lateral side of the shoe, because even the tiny part of a shoe, such as "heels", has been well shown to readers. The contact may refer to the woman in the poetry and readers outside the poetry, even though there is no real image about woman. It is a contact between the participant in poetry and bystanders outside the poetry. (3) The compositional metafunction is a whole view about poetry, similar to the textual function in discourse analysis. The three dimensions of information value, shooting a scene and prominence are the representation of devices applied by visual poets. Unlike the information value in traditional poetry (information above is ideal, and below is real), the information value in visual poetry may be indicated in the form of capital, punctuation and so forth. That "shoes" in the second vocabulary is capitalized as a representation of emphasis. Usually, the significant textual unit is in foreground, the others are in background, usually in the form of white space. The poet shoots the scene from the lateral side so that the shape of shoe with deep pink and dark pink colors is the foreground and the white space is the background.

In some extent, this visual poetry is a combination of constructivist and expressionist. For one thing, in the first sight, the poetry is like a shape of high-heeled shoe constructed by vocabularies, phrases and sentences. For another thing, it is also an intuitive structure in readers' minds. This visual poetry conveys the women's desperate aspiration for beauty. 


\section{The Analysis of Chinese Visual Poetry}

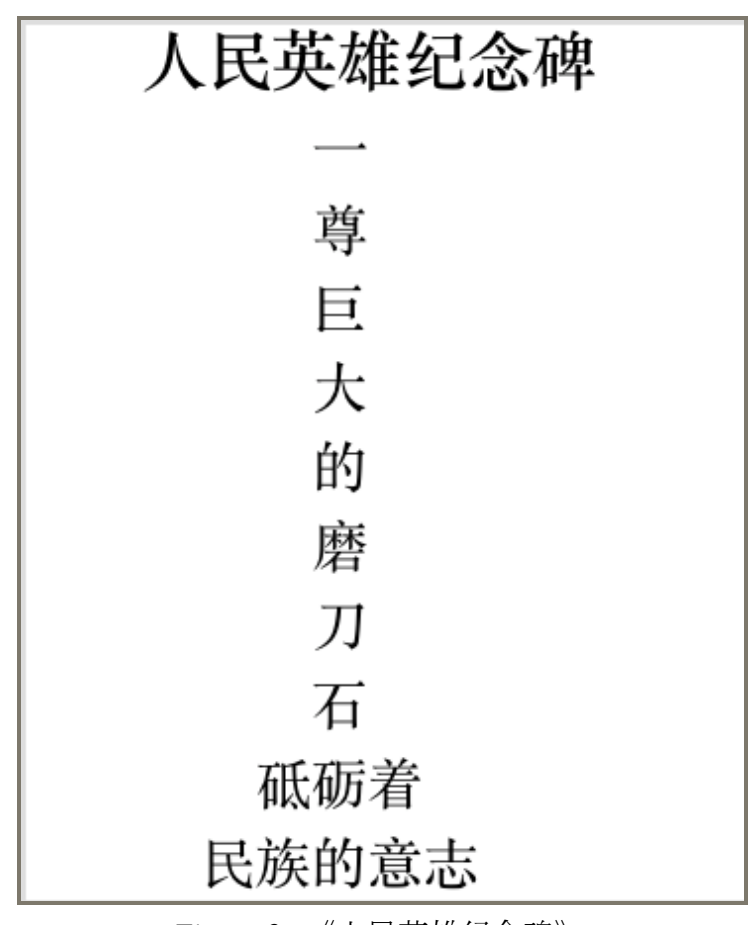

Figure 2. 《人民英雄纪念碑》.

This Chinese visual poetry can be translated into: Monument to the People’s Heroes: A great sharpening stone anneals the national volition.

Firstly, analyze the visual poetry from the traditional perspective. In the visual image, the shape of this Chinese visual poetry is like an enormous sharpening stone standing on the ground. The vocabularies “一尊巨大 的磨刀石 (A great sharpening stone)” are like a tremendous vertical stele. The vocabularies “砥砺着民族的意 志 (anneals the national volition)” are like the bottom of stele. In the verbal image, the vocabularies “磨刀石 (sharpening stone)” and “砥砺着 (anneals)” seem to convey the voice produced by knifes and swords that are grinding on the sharpening stone.

Secondly, from the perspective of multimodal discourse analysis based on the systematic functional theories, the analysis can be also developed from three dimensions: (1) The schema shaped by typographical device is obviously like the Monument of People's Heroes in China. The poet also applies the method of white space, leaving most space to be white, which seems to mean that the national volition stands perpetually and permanently in time and space. This Chinese visual poetry gives the readers an image and represents the conceptual function of the Monument of People's Heroes in China. (2) The schema in this Chinese visual poetry is presented in the way of distant shot from the frontage of the Monument of People's Heroes from the readers' perspective. The contact may refer to the Chinese national heroes in the poetry and readers, even though there is no real image of heroes. The relationship between the poet, the national heroes and readersis close, as this Chinese visual poetry conveys a spirit of strong national volition and strengthens the cohesiveness. (3) The information value in this Chinese visual poetry is mainly illustrated by the Chinese characters, because the first eight Chinese characters separately take one line independently. There are only 16 Chinese characters in the 
poetry, but each Chinese character represents its own information value, significantly and equally. The poet shoots the scene of Monument of People's Heroes from the frontage, which conveys the emotions of solemn and formality. The significant textual unit in the foreground is the image of the Monument of People's Heroes. The rest of the image is white space serving as background.

In this visual poetry, the expressionist is stronger than constructivist. Due to the only 16 Chinese characters, the visual poetry is not like the accumulation of vocabularies. However, the intuitive image of the Monument of People's Heroes appeared in readers' minds makes it expressionist. In a nutshell, this Chinese visual poetry illustrated tremendous respects and admiration for the Chinese national heroes, raising great resonance of readers.

\section{The Analysis of Visual Poetry Created by the Author}

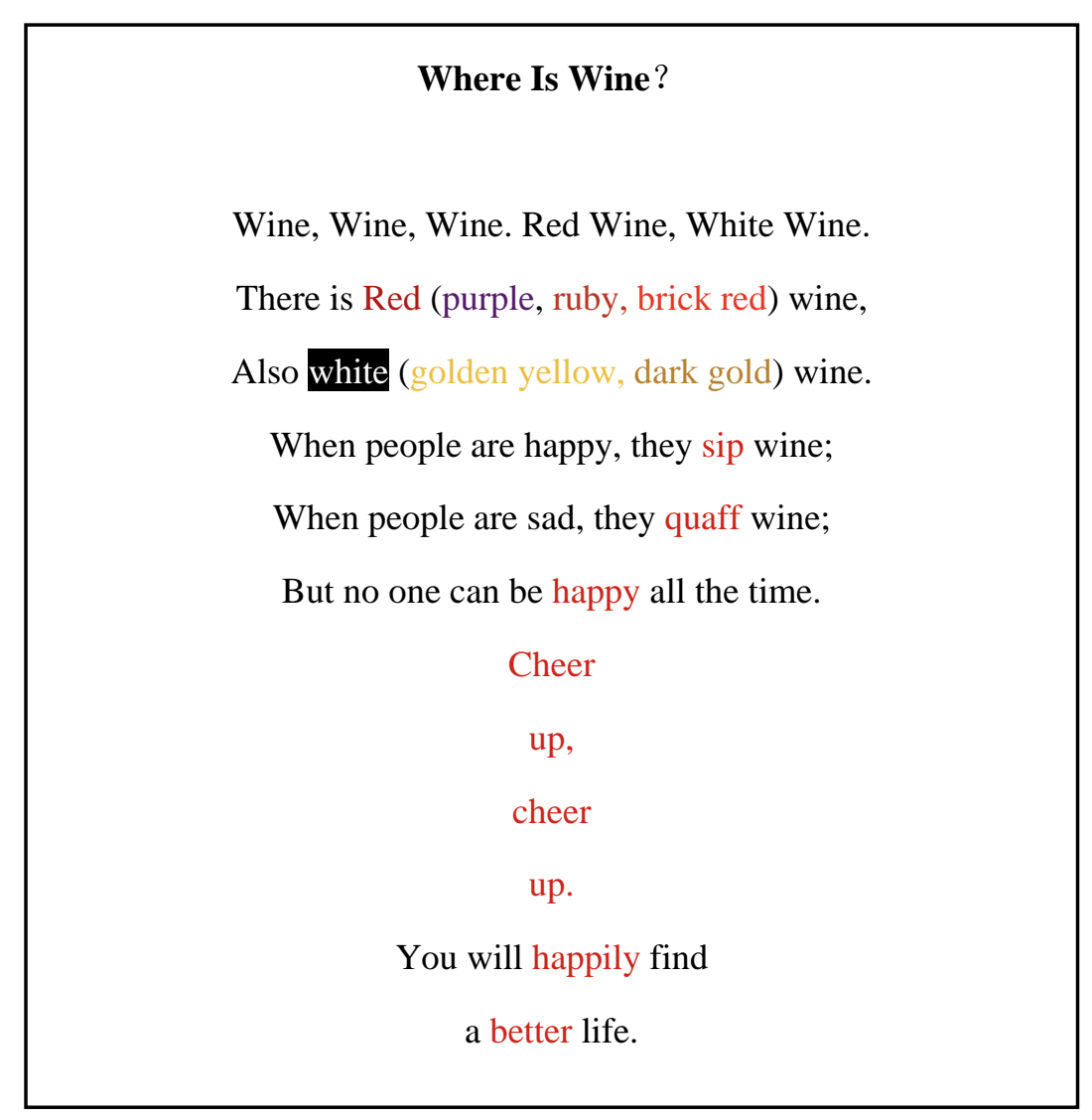

Figure 3. Where is wine?

Firstly, analyze the visual poetry from the traditional perspective. In the visual image, the shape of this visual poetry is like a goblet. As a goblet is composed of four parts, this visual poetry also consists of four parts. The fist and two lines "Wine, Wine. Redwine, white wine" are like the lip of a goblet. The shape of the three sentences "When happy, people sip wine. When sad, people quaff wine. No one can be happy all the time" are like the bowl of a goblet. "Cheer up, cheer up" is like the stem that connects the bowl and the foot of a goblet. The last sentence "You will find a better life" that is divided into two lines is like the foot of a goblet. Besides, the colors of vocabularies "red" and "white" are real red and white separately in the poetry, as well as "purple, ruby, 
brick red" and "golden yellow, dark gold", which are consistent with the meanings of vocabularies. In the verbal image, the sounds of the "wine" in the first four lines, the "time" in the fifth line, the "find" and the "life" in the last two lines make the whole visual poetry be of the characteristics of end rhyme and assonance.

Secondly, from the perspective of multimodal discourse analysis based on the systematic functional theories, the analysis can be also developed from three dimensions: (1) The title of this visual poetry is a question "Where is wine", which attracts readers' attention firstly to find where wine is. Then readers will find that the shape of the visual poetry by typographical device is like a goblet. It is obviously that wine is packed in the goblet. Now, an image of goblet that contains wine appears in readers' minds. Although the image of wine is uneasy to be described, the author applies the method of containment to describe the concept of wine and represent where wine is. It is worth noting that there is a question mark with different colors hidden in the poetry, which is an echo of the title. (2) From the readers' perspective, the shape in this visual poetry is presented in the way of close shot from any side of the goblet, due to its round feature. The contact refers to the people mentioned in the poetry and readers or common people, even though there is no real image of people. People in this poetry sip wine when happy and quaff wine when sad, just like people in ordinary life. (3) The information value in this visual poetry is different from that in the traditional poetry since it is a combination of poetry and painting. The author shoots the scene from any side of the goblet. The prominence of the visual poetry is the image composed of words, phrases and sentences. The white space is the background of the whole poetry.

Similarly, this visual poetry is a combination of constructivist and expressionist. For one thing, it is a shape of a goblet constructed by vocabularies, phrases and sentences. For another thing, it is also an intuitive structure in readers' minds combining the goblet in paper and a real goblet. Besides, the image of wine is contained in the goblet. The author tries to express that no one can go well all the time, but each one should cheer up and maintain positive attitudes towards life.

\section{Conclusion}

Visual poetry has both visual and verbal enjoyment for people, but the way of appreciation is quite different from each other. This research has focused on the three materials, Chinese visual poetry, western visual poetry and the visual poetry created by the author, applying the comparative method of traditional analysis method and the method based on multimodal theories. The new framework of analyzing the visual poetry has the three-dimensional structure: representation, interaction and composition. Each dimension has its own subclasses. In some extent, the constructivist and expressionist coexist together, contributing to expressing the thoughts and emotions of poets. In a nutshell, different genres represent different cultures, so the new framework provides an innovative perspective to analyze visual poetry and promotes an aesthetic experience to appreciate and enjoy Chinese and western visual poetry for ordinary people.

\section{References}

Bohn, W. (1986). The aesthetics of visual poetry 1914-1928. Chicago: The University of Chicago Press.

Jin, X. W. (2012). 卡明斯视觉诗多模态话语分析一以“in Just”为例. 海外英语, 19, 179-181.

Kress, G., \& Van Leeuwe, T. (2006). Reading images: The grammar of visual design. London: Routledge.

Li, X. L. (2010). 多模态话语分析视角下的视觉诗一以Goger McGough的诗歌“40-Love”为例. 延边教育学院学报, 4, 21-24. 
Li, Z. Z. (2003). 多模式话语的社会符号学分析. 外语研究, 5, 1.

Solt, M. E. (1968). Concrete poetry: A world view. Bloomington: Indiana University Press.

Tan, H. W. (2006). 漫谈西方视觉诗. 文艺理论与批评, 1, 133.

Wang, Y. H. (2009). 卡明斯诗歌“l(a”的多模态功能解读. 外语学刊, 9, 22-26.

Zhu, Y. S. (2007). 多模态话语分析的理论基础与研究方法. 外语学刊, 5, 82-86. 